\title{
The Emerging Role of the Hematopoietic Stem Cell Niche in Myeloproliferative Neoplasms
}

\author{
Biquan Luo* \\ Department of Pathology, Stanford University, Palo Alto, USA
}

Stanford Blood Center, Palo Alto, CA, USA

*Corresponding author:Biquan Luo, Department of Pathology, Stanford University, Palo Alto, CA, 94305 and Stanford Blood Center, Palo Alto, CA, 94304, USA, Tel: (650) 498-6134, E-mail: biquanl@stanford.edu

Received date: Aug 04, 2014; Accepted date: October 10, 2014; Published date: October 20, 2014

Copyright: (c) 2014 Luo B, This is an open-access article distributed under the terms of the Creative Commons Attribution License, which permits unrestricted use, distribution, and reproduction in any medium, provided the original author and source are credited.

\begin{abstract}
Myeloproliferative neoplasms (MPN) are chronic hematological neoplasms arising from the accumulation of genetic mutations in hematopoietic stem cells (HSCs). This notion is supported by genomic studies on MPN patients, in which recurrent mutations on genes such as JAK2, CALR, and MPL have been identified. Interestingly, recent studies using MPN mouse models have demonstrated that the HSC niche, where HSCs are located and maintained in the bone marrow (BM), plays an important role in MPN initiation and progression. Changes in the BM microenvironment alone can serve as a driver for altered hematopoiesis and hematological malignancies. Additionally, during MPN progression mutant HSCs remodel the HSC niche into a hospitable microenvironment for themselves that accelerates disease progression, creating a positive feedback loop where mutant HSCs thrive, and continue to alter the BM microenvironment to their advantage. In an attempt to provide a fresh perspective in understanding MPN pathogenesis, this review highlights the studies conducted that explore the role of the HSC niche in MPN pathogenesis and progression.
\end{abstract}

\section{Introduction}

Myeloproliferative neoplasms (MPN), previously known as myeloproliferative disorders (MPD), are chronic hematological neoplasms that are characterized by the excessive production of one or more subtypes of myeloid cells [1]. The myeloid cells involved in MPN, including red blood cells, platelets, and myeloid leukocytes such as granulocytes and monocytes, are derived from the hematopoietic stem cells (HSCs) and myeloid progenitors [2]. Thus, most studies on MPN have been concentrated on studying recurrent mutations in HSCs during the disease pathogenesis. Interestingly, HSCs are tightly regulated by both intrinsic gene expression and extrinsic cues from the bone marrow (BM) microenvironment [3], and recent studies on mouse models have shown that the latter may also play a role in MPN pathogenesis. The BM microenvironment refers to the stem cell niche in the BM where HSCs reside and their stemness maintained [4]. The HSC niche is composed of a variety of cells in the BM, including perivascular stromal cells (Nestin+ cells and Cxcl12-abundant reticular cells), sinusoidal endothelial cells, osteoprogenitors, osteoblast, adipocytes, and non-myelinating Schwann cells $[5,6]$. The vast majority of studies on MPN suggest that MPN development and progression is driven solely by genetic mutations in HSCs, and these mutations are believed to give autonomous proliferative advantage in certain lineages of blood cells [7]. However, several studies have recently suggested that along with HSC mutations, changes in the HSC niche is also an important regulator in MPN development and progression.

\section{MPN as a disease of mutations in HSCs}

A number of genomic studies examining MPN patients have identified recurrent somatic mutations that lead to constitutive activation of hematopoietic signaling pathways [8]. For example,
Philadelphia chromosome resulting from the translocation of ABL on chromosome 9 and BCR on chromosome 22 has been found in over $95 \%$ of chronic myeloid leukemia (CML) patients, being a most important criteria in the diagnosis of CML, a subgroup of MPN $[9,10]$. In the BCR-ABL negative MPN, JAK2 V617F is detected in approximately $95 \%$ of patients with polycythemia vera (PV), and $50-60 \%$ of essential thrombocythemia (ET) and primary myelofibrosis (PMF) patients [11,12]. Thrombopoietin receptor mutation MPL W515L/K has also been identified in 3\% of ET and $10 \%$ of PMF patients [7]. However, not all recurrent somatic mutations are directly tied to growth signaling pathways. Recently, a set of recurrent mutations on a gene encoding an endoplasmic reticulum chaperone, Calreticulin (CALR), have been identified in $25-27 \%$ of patients with ET and $14 \%-35 \%$ of patients with PMF [13-15]. Although the overexpression of mutant CALR resulted in elevated STAT5 activation [14], the function of CALR and its connection with JAK/STAT signaling has not been well characterized [7].

The functional validation of these mutations has been demonstrated in mouse models. For example, transplantation of BCR-ABL HSCs leads to CML and serves as a well-established mouse model for this disease [16]. Conversely, transgenic and knock-in mouse models that express JAK2 V617F in HSCs displayed various levels of MPN-like phenotypes [17]. To date, no studies have examined the effect of CALR mutations in the hematopoietic system on HSC physiology and MPN development, which could provide interesting insight into the role of this ER chaperone.

\section{The emerging role of HSC microenvironment in MPN progression}

As discussed above, most studies suggest that MPN is a clonal stem cell disease resulting from lesions on HSCs [18]. However, the intrinsic 
gene expression and the extrinsic microenvironment are two indispensible parts in regulating HSC activity. Therefore, this raises the question of whether the BM microenvironment plays a role in MPN progression.

In fact, an increasing number of studies have suggested that there is indeed an important role of the HSC niche in MPN development and progression. Mouse models have demonstrated that while genetic mutations are still believed to be the driver of hematological cancers, it is possible to induce MPN-like phenotypes or even leukemia by simply altering the BM microenvironment [19-21]. For example, retinoic acid receptor $\gamma(\operatorname{RAR} \gamma)$ is a highly conserved nuclear hormone receptor that acts as a ligand-dependent transcriptional regulator and regulates HSC cell fate [19]. RAR $\gamma$-deficient $\left(\mathrm{RAR} \gamma^{-/}\right)$mice developed a myeloproliferative syndrome with significantly elevated numbers of granulocytes in the $\mathrm{BM}$, spleen, and peripheral blood, and $\mathrm{BM}$ transplantation demonstrated that the effect solely resulted from the RAR $\gamma^{-1-}$ BM microenvironment [20]. More direct evidence came in 2010, where a targeted deletion of miRNA-processing endonuclease Dicer1 from osteoprogenitor cells resulted in phenotypes that recapitulated human myelodysplastic syndromes and secondary leukemia [21]. This study suggested for the first time that changes in the $\mathrm{BM}$ microenvironment alone can serve as a driver for altered hematopoiesis and even hematological malignancies (Figure 1).

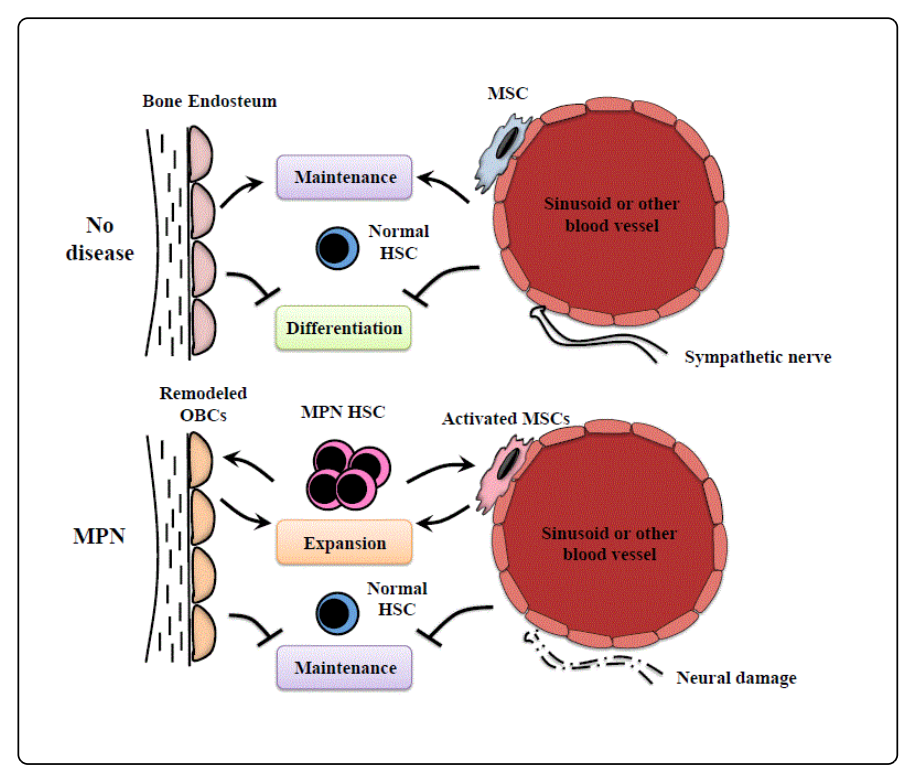

Figure 1: The positive feedback loop between the mutant HSCs and the HSC niche during MPN progression. Top panel: under normal conditions, the endosteal components and the endothelial components in the niche maintain HSCs and prevent differentiation; Bottom panel: during MPN progression, mutant HSCs remodel the niche by damaging its neural component, activating multipotent stromal cells (MSCs), and altering the supportive nature of osteoblastic lineage cells (OBCs). This niche remodeling turns the $\mathrm{BM}$ into a hospitable microenvironment that accelerates disease progression.

Altering the HSC niche is not only a potential disease-initiating step in the development of hematological disorders, but is also a mechanism to regulate the progression of existing diseases. For instance, BM transplantation recipients expressing constitutively activated parathyroid hormone receptor in osteoblastic cells displayed attenuated MPN phenotypes induced by $B C R-A B L$ positive HSCs when compared to the wild type recipients [22]. Interestingly, during MPN development, normal and mutant HSCs are believed to compete for the available niches, and mutant HSCs has been observed to remodel the function of the $\mathrm{BM}$ microenvironment to better suit disease progression. For example, in a BCR-ABL induced MPN model, the endothelial osteoblastic lineage cells isolated from MPN mice displayed an expansion with a concordant loss of supportive activity for wild type HSCs, whereas the MPN HSCs were insensitive to this loss of supportive activity, eventually outcompeting the wild type cells in BM transplantation assays [23]. Similarly, a recent study demonstrated that JAK2 V617F mutant HSCs produced excessive amount of interleukin- $1 \beta$ that triggered BM neural damage in the HSC niche. This neural damage was shown to be an essential process for MPN pathogenesis [24]. Taken together, these studies provided evidence for the presence of a vicious circle in MPN pathogenesis through interactions between mutant HSCs and the BM microenvironment.

\section{Conclusions and Perspective}

While most studies on MPN have been concentrated on genetic mutations in HSCs, emerging studies now present evidence that the HSC niche also has an important role in MPN pathogenesis. Under physiological conditions, the HSC niche maintains HSCs quiescence and prevents differentiation. However, this function of the HSC niche is compromised during MPN pathogenesis. The change of the BM microenvironment plays an essential role in advancing MPN disease progression, and the disease progression in return reinforces the microenvironment remodeling and thereby further tilting the already imbalanced hematopoiesis towards generating more dysregulated myeloid cells. With the active involvement of the HSC niche, the progression of MPN is no longer merely the accumulation of genetic mutations, but a positive feedback loop directly involving mutant HSCs and multiple BM components.

Despite multiple studies suggest that MPN progression can be driven through the HSC and niche feedback loop, there are many unanswered questions surround the specific role of HSC niche dysregulation in MPN. For instance, is the altered niche a cause or a consequence of the disease? Also, is the HSC niche dysregulation a stand-alone driver of the disease, an essential step in its progression, or a facilitating accelerator? It is also unknown which niche components participate in the remodeling process, and what their individual roles are. Studying these questions along with many others will begin to provide insight into this complicated disease process. The answers may vary depending on the biological context and more investigation of these queries is needed.

Moreover, a bigger question is whether the results on HSC niche in MPN obtained in mice can be translated to humans. While the genetic mutations identified in MPN patients can be feasibly validated for causal effects in mouse models, genetically modifying the HSC niche in healthy human or MPN patients is impractical. Targeting the HSC niche through pharmacological intervention may represent a viable option for studying the role of BM microenvironment in MPN pathogenesis, and ultimately uncover a new therapeutic option for MPN treatments. However, this needs to be based on a thorough understanding of the individual compartments in the HSC niche, their contributions to MPN development, and the signaling pathways that mediate interactions between the MPN HSC niche and stem cells. In 
Citation: Luo B (2014) The Emerging Role of the Hematopoietic Stem Cell Niche in Myeloproliferative Neoplasms. J Mol Genet Med 8: 133. doi: $10.4172 / 1747-0862.1000133$

Page 3 of 3

summary, recent studies suggest an important role exists for the HSC niche in the progression of MPN. Learning how HSCs and the niche are altered to promote MPN progression will be an important step to understanding and treating this disease.

\section{Acknowledgement}

I would like to thank Dr. Gregor Adams, Dr. James Zehnder, Dr. Jason Gotlib, Dr. Kyle Pfaffenbach, for their helpful discussions on the manuscript, and their guidance and generous support in my scientific training; thank Syed A Abidi for the helpful discussions on the manuscript.

\section{References}

1. Tefferi A, Thiele J, Vardiman JW (2009) "The 2008 World Health Organization classification system for myeloproliferative neoplasms: order out of chaos.," Cancer 115: 3842-3847.

2. Weissman IL, Shizuru JA (2008). "The origins of the identification and isolation of hematopoietic stem cells, and their capability to induce donor-specific transplantation tolerance and treat autoimmune diseases," Blood, 112: 3543-3553.

3. Zon LI (2008) Intrinsic and extrinsic control of haematopoietic stem-cell self-renewal. Nature 453: 306-313.

4. Adams GB, Scadden DT (2006) The hematopoietic stem cell in its place. Nat Immunol 7: 333-337.

5. Ugarte F, Forsberg EC (2013) Haematopoietic stem cell niches: new insights inspire new questions. EMBO J 32: 2535-2547.

6. Morrison SJ, Scadden DT (2014) The bone marrow niche for haematopoietic stem cells. Nature 505: 327-334.

7. Viny AD, Levine RL (2014) Genetics of myeloproliferative neoplasms. Cancer J 20: 61-65.

8. Tefferi A, Skoda R, Vardiman JW (2009) Myeloproliferative neoplasms contemporary diagnosis using histology and genetics. Nat Rev Clin Oncol 6: 627-637.

9. Onida F, Ball G, Kantarjian HM, Smith TL, Glassmanet A et al. (2002), "Characteristics and outcome of patients with Philadelphia chromosome negative, bcr/abl negative chronic myelogenous leukemia.," Cancer 95: 1673-84.

10. Zhang Y , J Rowley (2011) "Chronic Myeloid Leukemia?: Current Perspectives," Clin Lab Med: 5-8.
11. Tefferi A, Vainchenker W (2011). "Myeloproliferative neoplasms: molecular pathophysiology, essential clinical understanding, and treatment strategies.," J Clin Oncol, vol 29: 573-582.

12. Oh ST, Gotlib J (2010) JAK2 V617F and beyond: role of genetics and aberrant signaling in the pathogenesis of myeloproliferative neoplasms. Expert Rev Hematol 3: 323-337.

13. Tefferi A, Pardanani A (2014) Genetics: CALR mutations and a new diagnostic algorithm for MPN. Nat Rev Clin Oncol 11: 125-126.

14. Klampfl T, Gisslinger H, Harutyunyan AS, Nivarthi H, Rumi E et al. (2013), "Somatic mutations of calreticulin in myeloproliferative neoplasms.," N Engl J Med: 369:2379-2390.

15. Nangalia J, Massie CE, Baxter EJ, Nice FL, Gundem G, et al. (2013) "Somatic CALR mutations in myeloproliferative neoplasms with nonmutated JAK2.," N Engl J Med: 369, 2391-405.

16. Wertheim JA, Miller JP, Xu L, He Y, Pear WS (2002) The biology of chronic myelogenous leukemia:mouse models and cell adhesion. Oncogene 21: 8612-8628.

17. Li J, Kent DG, Chen E, Green AR (2011) Mouse models of myeloproliferative neoplasms: JAK of all grades. Dis Model Mech 4: 311-317.

18. HC Hasselbalch (2013), "Chronic inflammation as a promotor of mutagenesis in essential thrombocythemia, polycythemia vera and myelofibrosis. A human inflammation model for cancer development?," Leuk. Res: 37: 214-220.

19. Purton LE (2007) Roles of retinoids and retinoic Acid receptors in the regulation of hematopoietic stem cell self-renewal and differentiation. PPAR Res 2007: 87934.

20. Walkley CR, Olsen GH, Dworkin S, Fabb SA, Swann J, et al. (2007) A microenvironment-induced myeloproliferative syndrome caused by retinoic acid receptor gamma deficiency. Cell 129: 1097-1110.

21. Raaijmakers MH, Mukherjee S, Guo S, Zhang S, Kobayashi T, et al. (2010) Bone progenitor dysfunction induces myelodysplasia and secondary leukaemia. Nature 464: 852-857.

22. Krause DS, Fulzele K, Catic A, Sun CC, Dombkowski D, et al. (2013) Differential regulation of myeloid leukemias by the bone marrow microenvironment. Nat Med 19: 1513-1517.

23. Schepers K, Pietras EM, Reynaud D, Flach J, Binnewies M, et al. (2013) Myeloproliferative neoplasia remodels the endosteal bone marrow niche into a self-reinforcing leukemic niche. Cell Stem Cell 13: 285-299.

24. Arranz L, Sánchez-Aguilera A, Martín-Pérez D, Isern J, Langa X, et al. (2014) Neuropathy of haematopoietic stem cell niche is essential for myeloproliferative neoplasms. Nature 512: 78-81. 